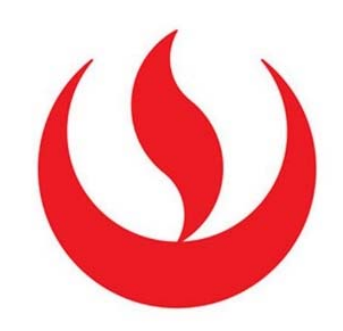

UNIVERSIDAD PERUANA DE CIENCIAS APLICADAS

Facultad de Ciencias de la Salud

Carrera de Terapia Física y Rehabilitación

\title{
ESTUDIO PILOTO: EFECTOS DE UN PROGRAMA DE PROPIOCEPCIÓN PARA AUMENTAR EL BALANCE DINÁMICO EN DEPORTISTAS UNIVERSITARIOS
}

\section{TESIS}

Para obtar por el grado de: Licenciado en Tecnología Médica con especialidad en Terapia Física y Rehabilitación

\author{
AUTORES \\ Enrique Abelino Zamora Gonzales (0000-0002-6738-6708) \\ Rodrigo Jesús Palza Linares (0000-0002-9923-4573)
}

Heidi Kosakowski (000-0003-3007-0803)

Giancarlo Becerra (0000-0002-7823-9682)

Lima, 6 de diciembre del 2018 


\section{Resumen del Documento}

El balance dinámico es una habilidad con relevancia en el deporte, ya que permite una mejor evolución del performance deportivo y evita el riesgo de lesiones. Asimismo, la propiocepción tiene un rol importante para el desarrollo del balance dinámico. Diseño de estudio: Se realizó un pre-experimental para evaluar el efecto de un programa de propiocepción sobre el balance dinámico en deportistas universitarios de futsal y básquet (damas y varones) que tengan entre 18 a 25 años de edad y pertenezcan a la Universidad Peruana de Ciencias Aplicadas. Para evaluar el balance dinámico se usó la herramienta Star Excursion Balance Test (SEBT) y un programa de propioceptivo que se llevó a cabo en un periodo de 2 semanas, 3 veces por semana con una duración de 15 a 25 minutos por sesión. Resultados : Se encontró significancia $(\mathrm{p}=0.034)$ entre el programa de propiocepción y el balance dinámico. Sin embargo, los participantes que asistieron menos de 3 sesiones de la intervención y los que entrenaban más de 10 horas semanales no se halló significancia. Conclusión : El programa de propiocepción tuvo un incremento en el balance dinámico en un $4.4 \%$.

Palabras clave : Propiocepción ; Balance dinámico ; Atletas universitarios ; Ejercicios propioceptivos. 


\section{ABSTRACT}

Dynamic balance is a skill that has relevance in sports, promoting better performance and reducing the risk of injuries. Moreover, proprioception has an important role for the development of the dynamic balance. A pre-experimental study was performed that assessed the effects of a proprioception program on dynamic balance. Subjects : university student athletes who played futsal and basketball (men and women), aged 18 to 25 years old, and enrolled at the Universidad Peruana de Ciencias Aplicadas in 2018. The Star Excursion Balance Test (SEBT) was used to assess dymanic balance. A proprioception program consisting of excericses that challeged dynamic balance twice a week for three weeks, 15 to 25 minutes per session was implemented. Results : Significance were found $(p=0.034)$ between the proprioception program and the dynamic balance. However, the participants who didn't attended less than three days of the intervention and who practice more than 10 hours per week didn't show significance. Conclussion: The proprioception program has an improve in the dynamic balance in $4.4 \%$.

Keywords: Proprioception, Dynamic balance, college athletes, proprioception exercises. 


\section{Tabla de Contenidos}

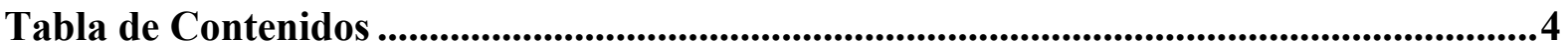

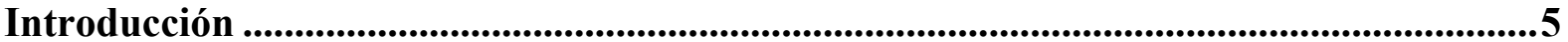

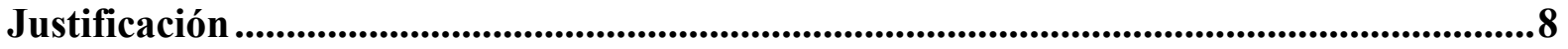

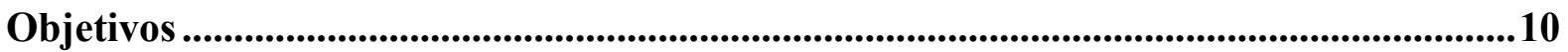

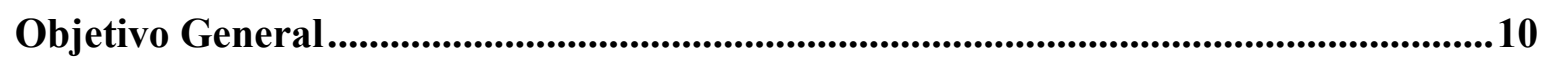

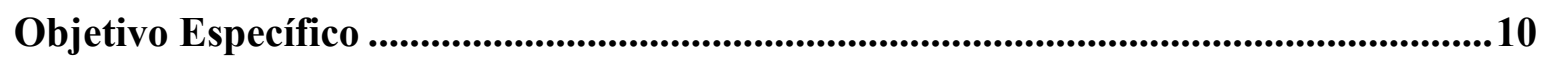

Hipótesis........................................................................................................................................11

Diseño y Lugar de estudio ..................................................................................................................12

Población de Estudio .............................................................................................................................13

Operalización de Variables ................................................................................................14

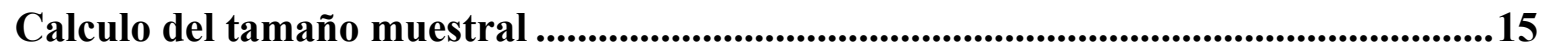

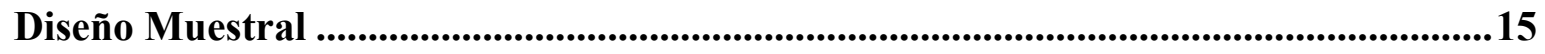

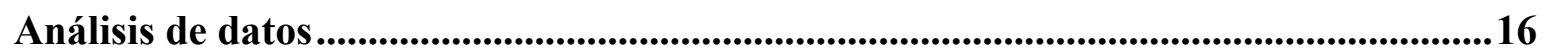

Procedimiento de recolección de Datos.....................................................................16

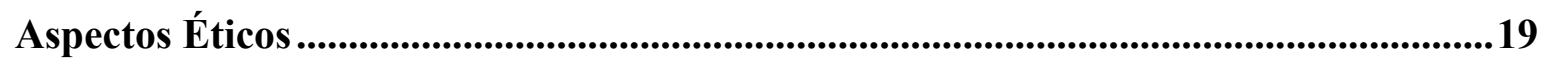

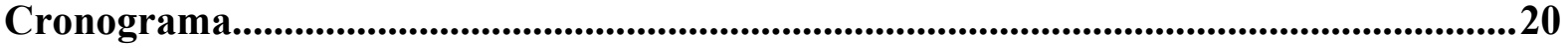

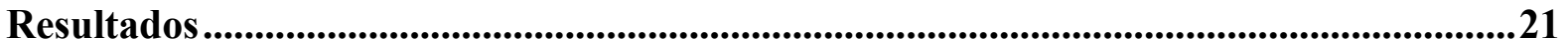

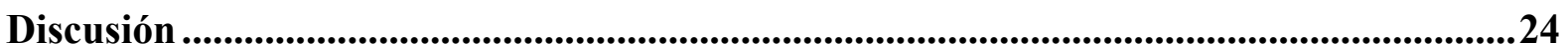

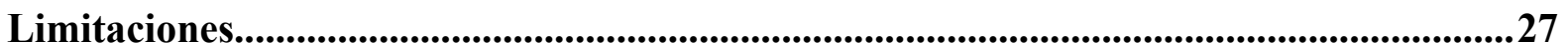

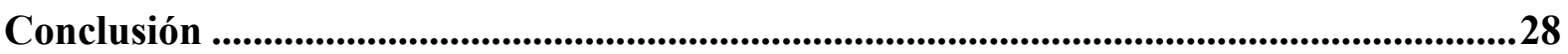

Referencias Bibliográficas................................................................................................29

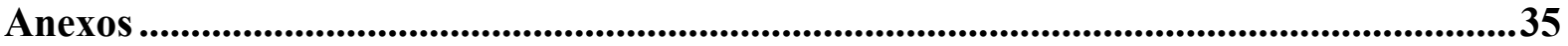




\section{Índice de Tablas}

Tabla 1-. Datos demográficos que muestra la media (X) y la desviación estandar (DE) de cada variable según los participantes.....................................................................................21

Tabla 2.- Comparación de pre y post intervención, según las variables : sexo (masculino y femenino), asistencia de menor a 3 sesiones y de mayor a 3 sesiones de la intervención, horas de prácticas semanales de mayor a 10 horas y de menor o igual a 10 horas, años de práctica del deporte mayor a 10 años y menor o igual a 10 años. (Media \pm desviación estandar) .....22 


\section{Introducción}

El balance dinámico es definido como la habilidad de mantenerse estable frente a diferentes tareas que se realizan durante el movimiento $(1,2)$. El déficit del balance dinámico en deportistas puede restrinjir su participación en diversas competencias (3). En esta población, el $80 \%$ de lesiones se da en tejidos blandos: músculos, ligamentos, tendones y articulaciones (4), teniendo un $13,4 \%$ de estas lesiones en miembros superiores, y un $67,7 \%$ en miembros inferiores, siendo las estructuras más comprometidas de este último la rodilla $45,5 \%$ y el tobillo $9,8 \%(3,4)$. Además, la prevalencia de lesiones en deportistas universitarios, sin importar el deporte específico que practican, es de un 53\%. (5).

Partiendo desde los más básico, el balance dinámico es la habilidad de mantener los segmentos de nuestro cuerpo estables, usando los recursos del sistema nervioso: propiocepción, sistema vestibular y las señales aferentes visuales, aportando información sensorio-motriz $(6,7,8)$. Esta habilidad tiene dos ejes principales, el balance estático y balance dinámico (6). El balance estático, es encargado de controlar la amplitud de oscilación del cuerpo con movimientos mínimos $(6,9)$. Mientras que, el balance dinámico se caracteriza por utilizar la información del entorno y del propio cuerpo para controlar y analizar los factores que intervienen en la estabilidad $(6,9)$. Se ha comprobado que el déficit del balance dinámico, trae consigo factores de riesgo en cuanto a lesiones deportivas de miembros inferiores; debido a que, al variar la estabilidad corporal se aumentan las fuerzas transmitidas a estructuras intra-articulares, ligamentosas y musculares, sobre todo en grupos estabilizadores grandes, como es el caso de los músculos isquiotibiales $(6,7)$. Además, se comprobó que el balance dinámico, es uno de los factores directamente implicados en el riesgo de lesiones de tobillo y rodilla en deportistas $(10,2)$. 
Se define propiocepción, como la percepción consciente e inconsciente del balance dinámico, estabilidad articular y sensación muscular, siendo responsable de la recopilación de las sensaciones relacionadas con la posición y movimiento de las articulaciones $(11,12,13)$; así como la función de integrar al sistema nervioso central los estímulos externos y los generados por el propio organismo $(14,15,13)$.

Existen solo algunos test específicos en la medición del balance dinámico, siendo el más utilizado, económico y el de mayor confianza el Star Excursion Balance Test (SEBT), también es útil en el registro de movilidad del tobillo, músculos intervinientes y rango de movimiento en la articulación de cadera (16). Gracias a lo antes mencionado, esta herramienta es sumamente utilizada, en diversos artículos destinados a prevención de lesiones musculo esqueléticas en deportistas, tanto amateur como profesionales $(16,13)$.

Por lo anterior señalado, se concluye la importancia de la propiocepción y el balance dinámico en deportistas; direcciona el objetivo de este estudio: determinar como la implementación de un programa de propiocepción, el cual está demostrado que no influye de manera negativa sobre la práctica deportiva, $(9,17)$, si no que influye favorablemente sobre el balance dinámico de deportistas amateurs universitarios. 


\section{Justificación}

En el contexto deportivo, sin interferir la práctica habitual de ejercicios durante el entrenamiento, cada atleta debe desarrollar la habilidad del balance dinámico, para conseguir el desarrollo de su desempeño y minimizar los riesgos de lesiones en miembros inferiores $(8,18,9)$; ya que, el balance dinámico constituye una base correcta para la realización de gestos deportivos complejos (2). La propiocepción posee un rol importante para potenciar el balance dinámico, ya que proporciona información sobre la postura corporal de manera integral $(19,12)$, siendo la propiocepción en la articulación de tobillo, la principal encargada de brindar mayor control en el balance dinámico en cualquier deporte $(8,20)$. En caso contrario, el défícit del balance dinámico, trae consigo un mayor riesgo de lesiones en miembro inferior, ya que aumenta las fuerzas transmitidas a estructuras intra-articulares, ligamentosas y musculares $(11,21)$. Debido a ello, es importante implementar un programa de propiocepción, ya que existe evidencia que el balance dinámico está significativamente relacionado con la mejora del performance y agilidad del deportista $(22,9,13,2)$.

Programas propioceptivos mejoraron los resultados en el balance dinámico en periodos de $5 \mathrm{a}$ 7 semanas $(16,9,12,13)$ y como complemento, existe también evidencia con respecto a que estos programas, pueden reducir la incidencia de lesiones en las extremidades inferiores, algunos tales como distensión de tobillo (13).

Con lo antes expuesto, y basándonos en antecedentes serios, en el presente estudio se decide registrar, como primer abordaje, el balance dinámico en las selecciones deportivas amateurs de la Universidad Peruana de Ciencias Aplicadas, el cual no se ha encontrado ningún estudio relacionado a este tema en Perú. Con referencia ante lo último expuesto, un estudio piloto preexperimental se acopla perfectamente en este contexto, debido a que es una investigación única en su naturaleza, refiriéndonos a la realidad nacional. Se consideran importantes los beneficios 
que soportan el estudio piloto de esta investigación, los cuales son: se proporciona una importante referencia para futuras investigaciones, detectando los principales problemas de manera anticipada; es de fácil ejecución que tolera poca inversión económica. Además, y por último, permite a los investigadores practicar y evaluar la efectividad de la recolección de datos y elaborar un correcto análisis estadístico (23). 


\section{Objetivos}

\section{Objetivo General}

El objetivo principal de este estudio es evaluar/medir el cambio del balance dinámico antes y después de la aplicación de un programa de propiocepción en deportistas universitarios de las selecciones de futsal y basquet de la Universidad Peruana de Ciencias Aplicadas.

\section{Objetivo Específico}

Evaluar el balance dinámico con la ayuda de la herramienta del Star Excursion Balance Test (SEBT).

Evaluar la diferencia del balance dinámico en las diferentes disciplinas deportivas en cuanto al sexo, edad, tiempo en horas de entrenamiento a la semana y años que practica el deporte.

Evaluar la diferencia del balance dinámico de los participantes que fueron más de 3 sesiones y los que asistieron menos de 3 sesiones.

Evaluar la diferencia del balance dinámico de los participantes que entrenan más de 10 horas semanales y menos de 10 horas semanales.

Evaluar la diferencia del balance dinámico de los participantes que practican el deporte más de 10 años y los que practican menos de 10 años. 


\section{Hipótesis}

El programa de propiocepción aumenta el balance dinámico en los deportistas universitarios de la Universidad Peruana de Ciencias Aplicadas. 


\section{Diseño y Lugar de estudio}

Se realizó un estudio pre-experimental, donde se llevó cabo un programa de propiocepción a los deportistas en las disciplinas de futsal damas y básquet varones, midiendo la variable de interés (balance dinámico) antes y después de la intervención. Se estudió el efecto de este programa sobre el balance dinámico, utilizando como herramienta de evaluación el Star Excursión Balance Test (SEBT).

El proceso de investigación consistió en evaluar en una fecha determinada el balance dinámico de todos los atletas de las selecciones deportivas ya mencionadas, con el SEBT. Una vez obtenido estos datos, se procedió a una doble digitación en tablas de Excel para luego proceder a un análisis estadístico. Después de haber evaluado el balance dinámico, se ejecutó el programa de propiocepción donde la intervención se llevó a cabo en 2 semanas (este programa se detallará en párrafos más adelante). Al término de las 2 semanas del programa de propiocepción, se evaluó nuevamente el balance dinámico en los participantes, esto con motivo de constatar los cambios en esta población.

Asimismo, el entrenamiento regular de los deportistas no infiere en el balance dinámico. Recientes investigaciones demuestran que los ejercicios de propiocepción son un complemento importante en el entrenamiento habitual de los atletas, debido a que mejora sus habilidades motoras (9). En otro estudio se menciona que el balance dinámico no está afectado por el ejercicio o actividad física, ni por el tiempo de práctica del deporte entre sujetos que practican el mismo deporte $(17,15)$. Por lo que, el entrenamiento habitual, no influirá en los resultados. 


\section{Población de Estudio}

Se aplicó la selección de deportistas que desearon participar en esta investigación y que pertenezcan a la Universidad Peruana de Ciencias Aplicadas, y además que integren las selecciones de futsal y básquet de la Universidad Peruana de Ciencias Aplicadas, en el periodo de abril y mayo del 2018. Los deportistas cumplieron los siguientes criterios de selección:

Criterios de Inclusión :

- Deportistas de 18 a 25 años de edad. (1)

- Ambos géneros

- Aceptar ser parte del estudio (CI)

Criterios de Exclusión :

- Deportistas con antecedentes quirúrgicos.

- Deportistas con lesiones actuales.

- Deportistas que no deseen participar del estudio.

- Deportistas que no asistan regularmente a los horarios de entrenamientos.

- Deportistas que estén realizando algún programa de recuperación.

- Deportistas que tomen AINES (Antiinflamatorios no esteroideos) (24) 


\section{Operalización de Variables}

Variables principales

\begin{tabular}{|l|l|c|c|c|c|}
\hline \multicolumn{1}{|c|}{ Variable } & Definición & Categorías & $\begin{array}{l}\text { Criterios de } \\
\text { medición de } \\
\text { las } \\
\text { categorías }\end{array}$ & $\begin{array}{c}\text { Tipos de } \\
\text { variables }\end{array}$ & $\begin{array}{l}\text { Escalas de } \\
\text { medición }\end{array}$ \\
\hline $\begin{array}{l}\text { Balance } \\
\text { dinámico } \\
\text { (variable } \\
\text { dependiente) }\end{array}$ & $\begin{array}{l}\text { capacidad de } \\
\text { poder mantenerte } \\
\text { en una posición } \\
\text { estable frente de } \\
\text { diferentes } \\
\text { estímulos (5) }\end{array}$ & porcentajes & SEBT & numéricas & Razón \\
\hline $\begin{array}{l}\text { Programa de } \\
\text { propiocepción } \\
\text { (variable } \\
\text { independiente) }\end{array}$ & $\begin{array}{l}\text { Estrategias para } \\
\text { mejorar las } \\
\text { habilidades } \\
\text { propioceptivas en } \\
\text { el atleta }\end{array}$ & $\begin{array}{l}\text { Nivel 1, } \\
\text { nivel 2, nivel } \\
\text { y nivel 4 }\end{array}$ & Abierta & Categórica & Ordinal \\
\hline
\end{tabular}

Variables Secundarias

\begin{tabular}{|l|l|l|l|l|l|}
\hline Variable & Definición & Categorías & $\begin{array}{l}\text { Criterios } \\
\text { de } \\
\text { medición } \\
\text { de } \\
\text { categorías }\end{array}$ & $\begin{array}{l}\text { Tipos de } \\
\text { variables }\end{array}$ & $\begin{array}{l}\text { Escalas } \\
\text { de } \\
\text { medición }\end{array}$ \\
\hline Edad & $\begin{array}{l}\text { Tiempo en años de } \\
\text { una persona desde } \\
\text { su nacimiento. }\end{array}$ & Años & Abierta & Numérica & Razón \\
\hline Peso & $\begin{array}{l}\text { Cantidad de masa } \\
\text { en la persona }\end{array}$ & $\begin{array}{l}\text { Deporte a que se } \\
\text { dedica } \\
\text { estudiante }\end{array}$ & Abierta & Numérica & Razón \\
\hline Deporte & $\begin{array}{l}\text { Género de la } \\
\text { persona }\end{array}$ & $\begin{array}{l}\text { Masculino, } \\
\text { femenino }\end{array}$ & abierta & Categórica & Nominal \\
\hline Sexo & & & & Categórica & Nominal \\
\hline
\end{tabular}




\begin{tabular}{|l|l|l|l|l|l|}
\hline Talla & $\begin{array}{l}\text { Altura del } \\
\text { participante en } \\
\text { centímetros }\end{array}$ & centímetros & Abierta & Numérica & Razón \\
\hline $\begin{array}{l}\text { Tiempo de } \\
\text { entrenamiento }\end{array}$ & $\begin{array}{l}\text { Tiempo } \\
\text { practica el deporte } \\
\text { durante el día }\end{array}$ & \# de horas & Abierta & Numérica & Razón \\
\hline $\begin{array}{l}\text { Tiempo práctica de } \\
\text { deporte }\end{array}$ & $\begin{array}{l}\text { Tiempo desde que } \\
\text { empezó la práctica } \\
\text { deportiva en la } \\
\text { disciplina a la que } \\
\text { se dedica ahora a } \\
\text { tiempo completo o } \\
\text { mayor frecuencia }\end{array}$ & Años & Abierta & Numérica & Ordinal \\
\hline
\end{tabular}

\section{Calculo del tamaño muestral}

Se usó el paquete estadístico Epidat ${ }^{\circledR}$ 4.2, donde se realizó una comparación de medias, según la bibliografía consultada, se espera lograr un cambio de 3.5\% en el Star Excursion Balance Test luego del programa de propiocepción; considerando una potencia del $80 \%$ y un nivel de confianza del $95 \%$ se obtuvo un resultado de 34 , de un solo grupo, por lo tanto será una muestra total de 68 deportistas de la Universidad Peruana de Ciencias Aplicadas.

\section{Diseño Muestral}

Se invitó a los deportistas de la Universidad Peruana de Ciencias Aplicadas que pertenecen a las selecciones de futsal damas y básquet varones. A todos los participantes que ingresaron a nuestro estudio se les informó sobre los objetivos de la investigación con un previo consentimiento informado. Las participantes tuvieron que pasar por los criterios de selección antes de ser evaluados. 


\section{Análisis de datos}

La recopilación de datos obtenidos en la encuesta y al SEBT fue tabulado e ingresado a un Excel. Se procedió a realizar una doble digitalización corroborar que no exista ningún error. Luego, la base de datos sin errores se ingresó al paquete estadístico Stata v11.0 donde se realizará las operaciones estadísticas correspondientes. Las variables categóricas se identificarán en proporciones y las variables numéricas se emplearán con media y desviación estandar. En primer lugar, usamos la operación estadística de Shapiro Wilk para corroborar la distribución normal de los datos. Dado que los datos muestran una distribución normal, continuamos con el $\mathrm{T}$ de student para las variables correspondientes.

\section{Procedimiento de recolección de Datos}

El proceso de recolección de datos empezó con una encuesta basal realizada por los evaluadores que cuenta con 13 preguntas sobre datos personales, edad, peso, talla, carrera profesional, tiempo de práctica del deporte, tiempo que entrena el deporte en la semana, si tuvo una lesión en los últimos 06 meses, si se ha sometido a proceso quirúrgico, si actualmente tiene una lesión, si actualmente está realizando un programa de propiocepción. Asimismo, se aplicó el Star Excursion Balance Test para medir el balance dinámico de los atletas participantes, para lo cual los evaluadores fueron asesorados para la realización correcta del test por un profesional de Terapia Física Deportiva y también se realizó un piloto de la evaluación con la finalidad de evitar sesgos en las evaluaciones.

\section{Protocolo Star Excursion Balance Test (SEBT):}

El Star Excursion Balance test, es un método para valorar el balance dinámico tanto como en deportistas y en personas que no realicen actividad física. Conocido también como el test de la estrella, consiste en 8 ejes (anterior, anterolateral, anteromedial, lateral, medial, posterolateral, posteromedial y posterior) (25). Actualmente, el SEBT se puede desarrollar de dos maneras: 
trazando las líneas en el piso y usando el kit del Star Excursion Balance Test, que es una herramienta fabricada (26).

El protocolo del SEBT empieza midiendo los miembros inferiores, desde la espina iliaca anterosuperior hasta el maléolo externo, este método tiene una confiabilidad de $\mathrm{ICC}=0,99$ (5). El participante deberá estar en parado en un solo pie (posición unipodal bípeda) en el punto donde se juntan los 8 ejes del SEBT. Asimismo, le pedimos al participante colocar sus dos manos sobre las crestas iliacas y luego le pedimos mantenese en apoyo unipodal y mover la extremidad inferior, que no se encuentra apoyada en el suelo, para alcanzar la máxima distancia que pueda en dirección a los 8 ejes correspondientes y luego retornar a su posición inicial.

Para valorar todo el procedimiento cuenta de 2 etapas: la primera, es darle al participante las pautas necesarias para realizar el correcto desarrollo del test, también, darle 2 intentos en cada eje para que se adecue, en esta etapa no se procederá a medir los resultados de los intentos. Luego de 2 minutos de descanso, la segunda etapa consiste en realizar 3 intentos para cada eje, en este caso si se medirá los resultados, para posteriormente tabularlos y sacar los resultados $(26,5,27)$.

Se invalidará los intentos si el participante transfiere la carga de peso a la extremidad inferior que realice el movimiento, si no mantiene estable sobre la extremidad inferior que se encuentra apoyada en el piso, si mueve la extremidad inferior que se encuentra apoyada en el piso, si falla al retorno de la extremidad inferior que realice el movimiento y, por último, si falla al mantener las manos sobre las crestas iliacas.

Una vez concluido el desarrollo del test, con las distancias en centímetros obtenidas del participante en cada eje del SEBT. Se procederá a obtener los porcentajes del balance dinámico de cada pierna según la distancia alcanzada y la medida de la extremidad inferior, con la siguiente fórmula:

$$
\%=\left(\frac{\text { Distanciaalcanzada }}{\text { Medidadelaextremidadinferior }}\right) \times 100
$$


Asimismo, se obtendrá el promedio en porcentajes del balance dinámico de cada pierna, sumando los 3 mejores resultados en centímetros y la medida de la extremidad inferior multiplicado 3 veces, con la siguiente fórmula:

$$
\%=\left(\frac{\text { Sumadelas } 3 \text { mejoresdistanciasalcanzadas }}{3 \times \text { Medidadelaextremidadinferior }}\right) \times 100
$$

Standing on LEFT limb

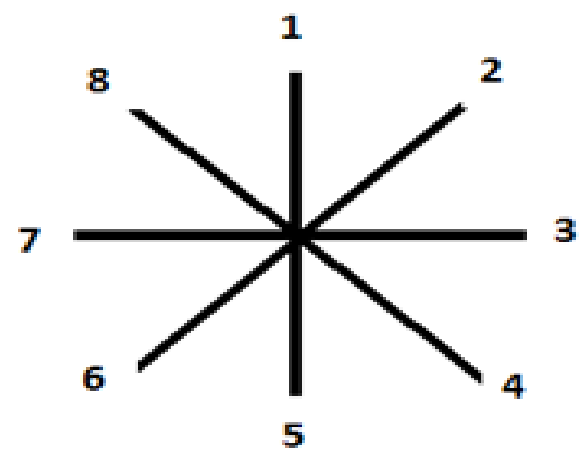

Standing on RIGHT limb

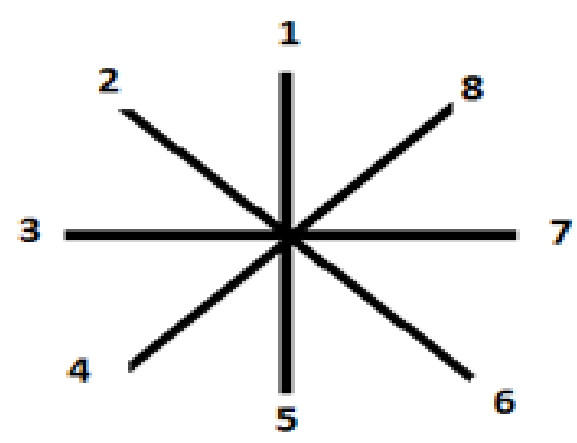

Diseño del Star Excursion Balance Test

El estudio se realizó en las instalaciones de la Universidad Peruana de Ciencias Aplicadas en el departamento de deportes, ubicado en el departamento de Lima, Perú. El desarrollo del programa y la evaluación pre y post intervención se llevó a cabo en abril y mayo del año 2018 .

\section{PROGRAMA DE PROPIOCEPCIÓN:}

Se realizó 2 semanas de entrenamiento propioceptivo; se dividirán los niveles por semana de aplicación del programa (28). Cada semana se aumentará el nivel de dificultad en los diferentes componentes y así poder manejar de manera eficiente las intensidades del programa.

Se realizarán 15- 20 minutos de ejercicios propioceptivos, luego del entrenamiento regular.

Se irá variando la intensidad del ejercicio en términos de: tiempo (duración de cada ejercicio y tiempo de descanso), información visual (Ojos abiertos o cerrados), tipo de superficie y planos de trabajo (Sagital, coronal o transversal). El programa de propiocepción se encuentra de manera más detalla en el Anexo 1 


\section{Aspectos Éticos}

El proyecto de tesis fue aprobado por el comité de ética de la Universidad peruana de Ciencias aplicadas (UPC). Además se obtuvo un permiso del Departamento de Deportes de la Universidad Peruana de Ciencias Aplicadas para poder acceder a su población. Debido a que el siguiente trabajo es pre-experimental, se hizo saber los detalles del programa de ejercicios a todos los participantes antes de empezar con su ejecución. Todos los datos recolectados durante las encuestas y los resultados del SEBT fueron totalmente confidenciales. 


\section{Cronograma}

\begin{tabular}{|c|c|c|c|c|c|c|c|c|c|c|}
\hline Actividad & 2017 & $\begin{array}{l}\text { Abril } \\
2018\end{array}$ & $\begin{array}{l}\text { Mayo } \\
2018 \\
\end{array}$ & $\begin{array}{l}\text { Junio } \\
2018 \\
\end{array}$ & $\begin{array}{l}\text { Julio } \\
2018 \\
\end{array}$ & $\begin{array}{l}\text { Agost } \\
2018\end{array}$ & $\begin{array}{l}\text { Sept } \\
2018 \\
\end{array}$ & $\begin{array}{l}\text { Oct } \\
2018\end{array}$ & $\begin{array}{l}\text { Nov } \\
2018\end{array}$ & $\begin{array}{l}\text { Dic } \\
2018 \\
\end{array}$ \\
\hline $\begin{array}{l}\text { Planteamiento } \\
\text { del problema de } \\
\text { investigación }\end{array}$ & & & & & & & & & & \\
\hline $\begin{array}{l}\text { Establecimiento } \\
\text { de la población y } \\
\text { tamaño de } \\
\text { muestra }\end{array}$ & & & & & & & & & & \\
\hline $\begin{array}{l}\text { Revisión ética del } \\
\text { protocolo }\end{array}$ & & & & & & & & & & \\
\hline $\begin{array}{lr}\text { Capacitación } & \\
\text { para } & \text { la } \\
\text { realización } & \text { del } \\
\text { SEBT y } & \text { el } \\
\text { programa } & \text { de } \\
\text { ejercicios } & \end{array}$ & & & & & & & & & & \\
\hline $\begin{array}{lr}\text { Intervención } & \\
\text { (SEBT } & \text { y } \\
\text { Programa } & \text { de } \\
\text { ejercicios) } & \end{array}$ & & & & & & & & & & \\
\hline Análisis de datos & & & & & & & & & & \\
\hline $\begin{array}{l}\text { Redacción de } \\
\text { tesis }\end{array}$ & & & & & & & & & & \\
\hline
\end{tabular}




\section{Resultados}

La tabla 1 (demográfica) muestra la media y la desviación estandar en las variables numéricas y en las variables categóricas se muestra la frecuencia y porcentaje. Se incluye, edad, peso, talla, deporte, tiempo de entrenamiento (en horas por semana), tiempo de práctica del deporte (en años).

Se recolectaron un total de 13 participantes, de los cuales 4 son varones y 9 son damas. Asimismo, 4 son deportistas masculinos de futsal y 5 deportistas femeninas de futsal y solo 4 deportistas femeninas de basquet.

Tabla 1-. Datos demográficos que muestra la media (X) y la desviación estandar (DE) de cada variable según los participantes.

\begin{tabular}{lccc} 
& & \multicolumn{2}{c}{$\mathrm{N}=13 * * *$} \\
\hline \multicolumn{1}{c}{ Variable } & $\mathrm{X}$ & $\mathrm{DE}$ \\
\hline Edad (años) & & 21 & 3 \\
Peso (kilogramos) & 63.62308 & 10.74742 \\
Talla (centímetros) & 163.0769 & 5.678344 \\
Tiempo de entrenamiento (horas & & 10.38462 & 4.42603 \\
a la semana) & & & \\
Tiempo de práctica (en años) & & & \\
Sexo $(*)$ & Masculino & 4 & $30.80 \%$ \\
& Femenino & 9 & $69.20 \%$ \\
Deporte $(* *)$ & & & \\
& Basquet & 4 & $30.80 \%$ \\
& Futsal & 9 & $69.20 \%$ \\
\hline
\end{tabular}

*En la variable sexo se muestra el total y el porcentaje que representa.

**En la variable deporte se muestra el total de participantes en cada disciplina deportiva y el porcentaje que representa.

$* * * \mathrm{~N}=$ total de participantes 
Tabla 2.- Comparación de pre y post intervención, según las variables : sexo (masculino y femenino), asistencia de menor a 3 sesiones y de mayor a 3 sesiones de la intervención, horas de prácticas semanales de mayor a 10 horas y de menor o igual a 10 horas, años de práctica del deporte mayor a 10 años y menor o igual a 10 años. (Media \pm desviación estandar)

\begin{tabular}{|c|c|c|c|c|c|}
\hline & Pre intervención $(*)$ & $\begin{array}{c}\text { Post } \\
\text { intervención }(*)\end{array}$ & Diferencia & $\begin{array}{c}\text { Intervalo de } \\
\text { confianza }\end{array}$ & $\mathrm{p}$ \\
\hline Futsal & $76.14 \pm 11.03$ & $80.04 \pm 7.75$ & 3.89 & {$[-2.00,9.80]$} & 0.166 \\
\hline Basquet & $78.99 \pm 7.61$ & $84.5 \pm 6.02$ & 5.50 & {$[1.25,9.75]$} & 0.025 \\
\hline Masculino & $74.35 \pm 12.94$ & $79.09 \pm 10.02$ & 4.74 & {$[-2.09,11.58]$} & 0.115 \\
\hline Femenino & $78.20 \pm 8.85$ & $82.44 \pm 6.25$ & 4.24 & {$[-1.48,9.96]$} & 0.126 \\
\hline $\begin{array}{l}\text { Asistieron }>3 \text { sesiones de } \\
\text { propiocepción. }\end{array}$ & $77.25 \pm 10.99$ & $81.70 \pm 8.76$ & 4.45 & {$[0.92,7.97]$} & 0.022 \\
\hline $\begin{array}{l}\text { Asistieron }<3 \text { sesiones de } \\
\text { propiocepción. }\end{array}$ & $76.82 \pm 9.70$ & $81.17 \pm 6.58$ & 4.35 & {$[-3.60,12.29]$} & 0.229 \\
\hline $\begin{array}{l}\text { Prácticas semanales }</=10 \text { horas } \\
\text { semanales }\end{array}$ & $79.67 \pm 9.85$ & $83.39 \pm 7.75$ & 3.72 & {$[0.99,6.44]$} & 0.013 \\
\hline $\begin{array}{l}\text { Prácticas semanales }>10 \text { horas } \\
\text { semanales }\end{array}$ & $71.06 \pm 7.93$ & $76.97 \pm 4.09$ & 5.91 & {$[-12.13,23.96]$} & 0.373 \\
\hline Deporte $</=$ a 10 años & $79.99 \pm 9.65$ & $83.02 \pm 6.99$ & 3.03 & {$[-0.27,6.33]$} & 0.667 \\
\hline Deporte $>10$ años & $72.26 \pm 9.14$ & $78.84 \pm 7.88$ & 6.58 & {$[-5.07,18.22]$} & 0.192 \\
\hline Total & $77.02 \pm 9.88$ & $81.42 \pm 7.33$ & 4.4 & {$[0.49,8.29]$} & 0.034 \\
\hline
\end{tabular}

\pm Desviación estándar

* Los resultados de pre y post intervención se muestran en porcentajes ajustado a la fórmula del SEBT. 
En la tabla 2 se presentan los resultados pre y post intervención del programa de propiocepción, separándolos en diferentes grupos para un mejor análisis. Los resultados pre y post intervención se presentan en porcentajes ajustados a la fórmula del SEBT. En primera instancia, se tiene un valor estadístico significativo en el grupo de basquet con un valor de $p=0.025$, lo que indica que hubo efecto y un cambio significativo con el programa de propiocepción a diferencia del grupo de futsal que obtuvo un valor de $\mathrm{p}=0.166$. Los participantes que recibieron más de 3 sesiones de propiocepción, obtuvieron un valor de $\mathrm{p}=0.022$, lo que se presume que mientras más días de propiocepción reciben los atletas mayor aumento del balance dinámico, a diferencia de los deportistas que solo fueron a tres sesiones o menos que obtuvieron un valor de $p=0.229$. Por otro lado, los participantes que entrenan menos de 10 horas semanales presentan un aumento de balance dinámico con un valor de $\mathrm{p}=0.013$ a comparación a los que entrenan más de 10 horas semanales. En un panorama y análisis general de todos los participantes se obtiene un valor estadísticamente significativo $(\mathrm{p}=0.034)$, lo que demuestra a grandes rasgos que el programa de propiocepción tiene relación con el aumento del balance dinámico en deportistas universitarios. 


\section{Discusión}

Muchas investigaciones se han hecho acerca de la propiocepción y como esta influye en el balance dinámico en los deportistas, especialmente, atletas universitarios. La finalidad de nuestra investigación fue evidenciar el cambio del balance dinámico a partir de nuestro programa de propiocepción en atletas de las selecciones de futsal (damas y varones) y de basquet (damas) de la Universidad Peruana de Ciencias Aplicadas en Lima, Peru. Y, también, dar soporte a las investigaciones ya realizadas, por lo tanto, llevarlas a un contexto nacional y obtener el mayor provecho. El programa de propiocepción que se brindó a los atletas de la Universidad Peruana de Ciencias Aplicadas aumentó su balance dinámico (pre-intervernción: $77.02 \pm 9.88$, post-intervención: $81.42 \pm 7.33$, tabla 2), como también lo soportan otras investigaciones (29,30,31). Además, el cambio mínimo clínico ajustado al Star Excursion Balance Test es de $3.0-4.6 \%$ (32), por lo que los resultados obtenidos fueron positivos, con un cambio del balance dínamico de un total de 4.4\% (tabla2). Este resultado soporta que nuestro programa de propiocepción aumenta el balance dinámico, como lo demuestra también Brandon M. Ness, Brett A. Comstock y William E. Schweinle que encontraron en su estudio una diferencia entre $3 \%$ y $7 \%(33)$.

Por otro lado, Butler, R. J. et. al. menciona en su estudio que los jugadores universitarios de futbol que poseen menos de un valor total (SEBT) de $89.6 \%$ tienen mayor probabilidad de tener una lesión en los miembros inferiores (34). Los jugadores de futsal, antes de la intervención obtuvieron un valor de 76.14\% ( \pm 11.03$)$ y, después de la intervención un valor de $80.04 \%$ ( \pm 7.75) con un auemnto del $3.89 \%$ (diferencia). Estos dos valores se encuentran debajo de $89.6 \%$, que según Butler, R. J, tienen mayor probabilidad de adquirir una lesión (34). Estos datos son muy importantes, ya que manifiesta en los jugadores de futsal y de basquet, que fueron incluidos en este estudio, tienen mayor probabilidad de adquirir una lesión en los miembros 
inferiores. Por lo tanto, aplicar nuestro programa de propiocepción mejorará el balance dinamico y así poder reducir el riesgo de lesiones en los jugadores.

Se pueden mejorar y añadir ciertos elementos estratégicos al programa de propiocepción a medida que se descubren nuevas mejoras en este tema, como es en el estudio de Leavey, V. J., Sandrey, M. A., \& Dahmer, G. (2010) que encontraron que, mientras más ejercicios para glúteo medio, añadido con los ejercicios de propiocepción, se obtienen mejores resultados en el balance dinámico (35).

Cabe resaltar que existe discrepancia en algunos resultados que se obtuvieron en esta investigación, como causalidad, la falta de población adecuada y la diferencia entre hombres y mujeres de cada deporte. Se encontró significancia en las deportistas de basquet, lo cual no hace ajeno ya que literaturas avalan el resultado obtenido (37). Por otro lado, los atletas de futsal, que participaron en nuestra investigación, no obtuvieron un resultado estadísticamente significativo; sin embargo, se encontró en otras investigaciones resultados positivos, contrarios a los nuestros $(29,36)$; por lo que, posiblemente, se presume por la falta de participantes en este deporte sea la causante de esta deficiencia.

Por otro lado, se encontró significancia en los participantes que acudieron más de tres sesiones de propiocepción, y no se presentó resultados positivos a los que asistieron menos de tres sesiones de propiocepción. El menor tiempo de entrenamiento propioceptivo que se encontró en otras investigaciones fue de 4 semanas (38). Por lo que se podría plantear como relevancia científica, para próximas investigaciones, obtener resultados positivos a partir más de 3 sesiones y/o de dos semanas de propiocepción.

Así también, se encontró una diferencia importante entre los participantes que acuden menos de 10 horas semanales versus a los que asisten más de 10 horas semanales a sus entrenamientos. Es un punto interesante, debido a que se podría presumir que los deportistas que más tiempo 
dedican a su disciplina deportiva tendrían más balance dinámico, sin embargo, debido a la cantidad de horas invertidas pueden sufrir de fatiga muscular, lo cual esta condición disminuye el balance dinámico (39). En adición, al momento de realizar el Star Excursion Balance test (SEBT) también se reproduce activación muscular empeorando la condición de fatiga y afectando el resultado del SEBT (40). 


\section{Limitaciones}

Es importante recalcar los inconvenientes que se presentaron en el proceso de la investigación. Debido a la construcción del polideportivo en el campus Villa, de la Universidad Peruana de Ciencias aplicadas, que se ejecutó en los meses de marzo, abril, mayo, junio y julio, las selecciones deportivas entrenaban en diferentes establecimientos de la univeridad y centros privados ajenos a nuestra institución, haciendo dificil el acceso para las evaluaciones. Asimismo, los horarios de los entrenamientos eran muy variados, desde la mañana, tardes-y noche.

Por otro lado, debido a que se empezó a recolectar los datos en los meses de abril y mayo, las selecciones deportivas de futsal y basquet se encontraban en la mitad del campeonato. Los entrenadores se enfocaban en desarrollar su estrategia de entrenamiento dejando de lado la intervensión de nuestra investigación $\mathrm{y}$, además, estas selecciones faltaban a días de entrenamiento por competir en sus respectivos partidos.

Para futuras investigaciones se recomienda tener en cuenta una comitiva entre los entrenadores de las selecciones deportivas, el coordinador y/o jefe del área de deportes y los investigadores, presentando el protocolo de intervención, junto con las fechas de evaluación y del programa propioceptivo. Además, incluir la confirmación de parte de los entrenadores para que se incluya el programa de propiocepción dentro del entrenamiento regular. Por otro lado, es importante brindar con total transparencia los beneficios para los deportistas y como influye esta habilidad dentro de su actividad deportiva. Se requiere tiempo y compromiso para emplear este tipo de investigación. 


\section{CONCLUSIÓN}

El SEBT como herramienta de medición es sencilla de elaborar y facilita al evaluador para obtener resultados coherentes y de importancia. Muchas herramientas de medición para el balance dinámico existen para la investigación como, por ejemplo: el Y-balance test ${ }^{44}$, que es una reducción del SEBT y que posee buenos resultados. Así como también el Dynamic Leap and Balance Test (DLBT) (41) que se deben tener en consideración en futuras investigaciones o comparar los resultados de estas herramientas de medición.

Los resultados obtenidos en el estudio fueron significativos, con un total de un aumento de 4.4\% que se encuentra dentro del cambio clínico significativo. Se sugiere aplicar los ejercicios de propiocepción en deportistas universitarios, sin embargo, la causa de la no asociación en algunos de los participantes es relativa. 


\section{Referencias Bibliográficas}

(1) Bhat, R. Moiz, J. Comparison of dynamic balance in collegiate field hockey and football players using Star Excursion Balance Test. Asian Journal of Sport Medicine. 2013. 4(3).

(2) Ricotti L. Static and dynamic balance in young athletes. J. Hum. Sport Exerc 2011;6(4): 616-628

(3) Zemvoká E. Sport-Specific Balance. Sports Med (2014) 44: 579.

(4) Osorio J, Clavijo M, Arango V, et al. g. IATREIA 2007; 20(2): 160-177

(5) Mikel R. Stiffler, David R. Bell, Jennifer L. Sanfilippo, et al. Star Excursion Balance Test Anterior Asymmetry is Associated with Injury Status in Division I Collegiate Athletes. Journal of Orthopaedic \& Sports Physical Therapy. 2017.

(6) Wang H, Ji Z, Jiang G, Liu W, Jiao X. Correlation among proprioception, muscle strength and balance. J Phys Ther Sci 2016; 28(12): 3468-3472.

(7) Fort A, Costa L, De Antolín P, Massó N. Efectos de un entrenamiento propioceptivo sobre la extremidad inferior en jóvenes deportistas jugadores de voleibol. Apunts. Medicina de 1'Esports 2008. Vol (43): 5-13.

(8) Jia Han, Judith Anson, Gordon Waddington, Roger Admas, Yu Liu. The Role of Ankle Proprioception for Balance Control in relation to Sports Performance and Injury. BioMed Research International. 2015.

(9) Con Hrysomallis. Balance Ability and Athletic Performance. Sports Med. 2011; 41(3): 221-232.

(10) Ueblacker P, Haensel L, Mueller-Wohlfahrt HW. Treatment of muscle injuries in football. J Sports Sci 2016; 34(24): 2329-2337 
(11) El-Gohary TM, Khlaed OA, Ibrahim SR, Alshenqiti AM, Ibrahim MI. Effect of proprioception cross training on repositioning accuracy and balance among healthy individuals 2016. J Phys Ther Sci; 28(11): 3178-3182

(12) Romero N, Martínez E, Lomas R, et al. Short-term Effects of proprioceptive training with unstable platform on athletes stabilometry.Journal of Strength and Conditioning Research 2013; 27(8): 2189-2197

(13) López-González L, et al. Prevención de esguinces de tobillo en jugadoras de baloncesto amateur mediante programas de propiocepción. Estudio piloto de casos y controles. Fisioterapia. 2014; 37(5): 212-22

(14) Cerulli M, Benoit L, Carafía A, Ponteggia F. Proprioceptive training and Prevention of Anterior Cruciate Ligament Injuries In Soccer. Journal of Orthopaedic \& Sport Physical Therapy 2001; 31(11): 655-660

(15) McCaskey M, Schuster-Amft C, Wirth B, Suica Z, D de Bruin E. Effects of proprioceptive exercises on pain and function in chronic neck- and low back pain rehabilitation: a systematic literature review. BMC Musculoskeletal Disorders 2014; 15: 382 .

(16) Fort A, Romero D, Costa L, Bagur C,Lloret M, Montañola A. Diferencias en la estabilidad postural estática y dinámica según el sexo y pierna dominante. Apunts Medicina de 1'Esport 2009; 44(162):74-81.

(17) Schneiders A.G., Sullivan S.J., Handcock P., Gray A., McCrory P.R. Sports concussion assessment: the effect of exercise on dynamic and static balance. Scandinavian Journal of Medicine \& Science in Sports. 2012(22); 85-90.

(18) J. Han, J. Anson, G. Waddington, R. Adams. Sport attainment and proprioception. International Journal of Sports Science And Coaching. 2014, 9(1): 159-170. 
(19) Ben Moussa Zouita, A., Majdoub, O., Ferchichi, H., Grandy, K., Dziri, C., \& Ben Salah, F. Z. (2013). The effect of 8-weeks proprioceptive exercise program in postural sway and isokinetic strength of ankle sprains of Tunisian athletes. Annals of Physical and Rehabilitation Medicine, 56(9-10), 634-643.

(20) Desphande N, Simonsick E, Metter E, et al. Ankle proprioceptive acuity is associated with objetive as well as self-report measures of balance,mobility, and physical function. American Aging Association 2016; 38:53

(21) Johansson AC, Karlsson H. The star excursion balance test: criterion and divergent validity on patients with femoroacetabular impingement. Man Ther 2016; 26: 2014109

(22) D. Sekulic, M. Spasic, D. Mirkov, M. Cavar, and T. Sattler. Gender-Specific influence of balance, speed, and power on agility performance. Journal of Strength and Conditioning Reseach. 2013, 27(3): 802-811.

(23) Doody, O., \& Doody, C. M. (2015). Conducting a pilot study: case study of a novice researcher. British Journal of Nursing, 24(21), 1074-1078.

(24) Tscholl P, Vaso M, Weber A, Dvorak J. High prevalence of medication use in professional football tournaments including the World Cups between 2002 and 2014: a narrative review with a focus on NSAIDs. Br J Sports Med 2015;49:580-582.

(25) Phillip A. Gribble, Jay Hertel. Considerations for Normalizing Measures of the Star Excursion Balance Test. Measurement in Physical Education and Exercise Science. 2003, 7(2): 89-100.

(26) Linek P, Sikora D, Wolny T, Saulicz E. Reliability and number of trials of Y balance Test in adolescent athletes. Musculoskeletal Science and Practice. (2017) 
(27) Phillip J. Plisky, Mitchell J. Rauh, Thomas W. Kaminski, Frank B. Underwood. Star Excursion Balance Test as a Predictor of Lower Extremity Injury in High School Basketball Players. Journal oh Orthopaedic \& Sports Physical Therapy. (2017)

(28) Chauntelle M, R. Keats M. Comparing the Effects of a Novel And Traditional Proprioceptive Balance Training Program on Activity Adherence and Balance Control in a Healthy University Population: A Preliminary Study. Games for Health Journal: Research, Development, and Clinical Applications. 2013:2(5).

(29) Filipa, A., Byrnes, R., Paterno, M. V., Myer, G. D., \& Hewett, T. E. (2010). Neuromuscular Training Improves Performance on the Star Excursion Balance Test in Young Female Athletes. Journal of Orthopaedic \& Sports Physical Therapy, 40(9), $551-558$.

(30) Cain, M. S., Garceau, S. W., \& Linens, S. W. (2017). Effects of a 4-Week Biomechanical Ankle Platform System Protocol on Balance in High School Athletes With Chronic Ankle Instability. Journal of Sport Rehabilitation, 26(1), 1-7.

(31)Cruz-Diaz, D., Lomas-Vega, R., Osuna-Pérez, M., Contreras, F., \& Martínez-Amat, A. (2015). Effects of 6 Weeks of Balance Training on Chronic Ankle Instability in Athletes: A Randomized Controlled Trial. International Journal of Sports Medicine, 36(09), 754-760.

(32) Fullam, K., Caulfield, B., Coughlan, G.F., \& Delahunt, E. (2014). Kinematic Analysis of Selected Reach Directions of the Star Excursion Balance Test Compared with the Y-Balance Test. Journal of Sport Rehabilitation, 23(1), 27-35.

(33) Brandom M, et al. Changes in dynamic balance and hip strength after an eight week conditioning program in NCAA division I Female soccer athletes. The international journal of sports physical therapy. 2016. Vol11(7).1054 
(34) Butler, R. J., Lehr, M. E., Fink, M. L., Kiesel, K. B., \& Plisky, P. J. (2013). Dynamic Balance Performance and Noncontact Lower Extremity Injury in College Football Players. Sports Health: A Multidisciplinary Approach, 5(5), 417-422.

(35) Leavey, V. J., Sandrey, M. A., \& Dahmer, G. (2010). Comparative Effects of 6-Week Balance, gluteus Medius Strength, and Combined Programs on Dynamic Postural Control. Journal of Sport Rehabilitation, 19(3), 268-287.

(36) Butler, R. J., Southers, C., Gorman, P. P., Kiesel, K. B., \& Plisky, P. J. (2012). Differences in Soccer Players’ Dynamic Balance Across Levels of Competition. Journal of Athletic Training, 47(6), 616-620.

(37) Pfile, K. R., Gribble, P. A., Buskirk, G. E., Meserth, S. M., \& Pietrosimone, B. G. (2016). Sustained Improvements in Dynamic Balance and Landing Mechanics After a 6-Week Neuromuscular Training Program in College Women's Basketball Players. Journal of Sport Rehabilitation, 25(3), 233-240.

(38) Melong, C., \& Keats, M. R. (2013). Comparing the Effects of a Novel and a Traditional Proprioceptive Balance Training Program on Activity Adherence and Balance Control in a Healthy University Population: A Preliminary Study. Games for Health Journal, 2(5), 308-312.

(39) Hosseinimehr. (2010). The Effects of Fatigue and Chronic Ankle Instability on Dynamic Postural Control. Physics International, 1(1), 22-26.

(40) Earl, J. E., \& Hertel, J. (2001). Lower-Extremity Muscle Activation during the Star Excursion Balance Tests. Journal of Sport Rehabilitation, 10(2), 93-104.

(41) Jaffri, A. H., Newman, T. M., Smith, B. I., \& John Miller, S. (2017). THE DYNAMIC LEAP AND BALANCE TEST (DLBT): A TEST-RETEST RELIABILITY STUDY. International Journal of Sports Physical Therapy, 12(4), 512-519. 
(42) Verhagen E, Van der Beek A, Twisk J, et al. The effect of a Proprioceptive Balance Board Training Program for the Prevention of Ankle Sprains. The American Journal of Sports Medicine 2004; 32(6): 1385-1393.

(43) Eils E, Rosenbaum D. A multi-station proprioceptive exercise program in patients with ankle instability. Med. Sci. Sports. Exerc. 2001; 33 (12): 1991-1998. 


\section{Anexos}

ANEXO 1

EJERCICIOS $(12,42,43,13)$

SIN MATERIALES

- Apoyo unipodal (Flexión de cadera)

- Apoyo unipodal (extensión de cadera)

- Apoyo unipodal (flexión de tronco y abducción de brazos)

CON BALÓN (Balón de futsal, basquet)

- Lanzamiento de balón con apoyo unipodal y flexión de rodilla

- Lanzamiento de balón con apoyo unipodal y flexión de rodilla y cadera

\section{CON SUPERFICIES INESTABLES Y BALÓN}

- Bípedo sobre superficie inestable

- Apoyo unipodal sobre superficie inestable

- Bípedo sobre superficie inestable y lanzamiento de balón

- Apoyo unipodal sobre superficie inestable y lanzamiento de balón

Los ejercicios se realizarán con una frecuencia de 3 veces por semana durante 2 semanas.

Durante el desarrollo de las 6 sesiones de ejercicios de propiocepción se irá incrementando el nivel de dificultad de los mismos; para lo cual, se hará uso de superficies inestables y se le pedirá a los deportistas cerrar los ojos durante algunos de los ejercicios. 
SESIONES 1 Y 2 (NIVEL 1)

\begin{tabular}{|c|c|c|c|c|}
\hline EJERCICIOS & DESCRIPCIÓN & TIEMPO & $\begin{array}{l}\text { INFORMACIÓN } \\
\text { VISUAL }\end{array}$ & $\begin{array}{c}\text { TIPO DE } \\
\text { SUPERFI } \\
\text { CIE }\end{array}$ \\
\hline $\begin{array}{l}\text { Apoyo unipodal } \\
\text { (Flexión de } \\
\text { cadera) (42) }\end{array}$ & $\begin{array}{l}\text { El deportista } \\
\text { mantiene la posición } \\
\text { sobre un pie y } \\
\text { rodilla extendida y } \\
\text { con la pierna } \\
\text { contralateral realiza } \\
\text { flexiones de cadera }\end{array}$ & $\begin{array}{l}\text { - Mantiene la } \\
\text { posición y realiza } \\
\text { el movimiento de } \\
\text { flexión durante } 45 \\
\text { segundos por cada } \\
\text { lado } \\
2 \text { Series por cada } \\
\text { pierna }\end{array}$ & Ojos abiertos & $\begin{array}{l}\text { Plana } \\
\text { regular }\end{array}$ \\
\hline $\begin{array}{l}\text { Apoyo unipodal } \\
\text { (Extensión de } \\
\text { cadera) (42) }\end{array}$ & $\begin{array}{l}\text { El deportista } \\
\text { mantiene la posición } \\
\text { con apoyo de un } \\
\text { solo pie y con el } \\
\text { miembro } \\
\text { contralateral realiza } \\
\text { una extensión de } \\
\text { cadera }\end{array}$ & $\begin{array}{l}\text { Mantiene la } \\
\text { posición y realiza } \\
\text { el movimiento } \\
\text { durante } \\
\text { segundos } \\
25 \\
2 \text { Series por cada } \\
\text { pierna }\end{array}$ & Ojos abiertos & $\begin{array}{l}\text { Plana- } \\
\text { regular }\end{array}$ \\
\hline $\begin{array}{l}\text { Apoyo unipodal } \\
\text { (Flexión de } \\
\text { cadera) } \\
\text { Recepción del } \\
\text { balón } \\
(42)\end{array}$ & $\begin{array}{l}\text { El deportista } \\
\text { mantiene el apoyo } \\
\text { unipodal y con la } \\
\text { rodilla extendida y } \\
\text { con la pierna } \\
\text { contralateral realiza } \\
\text { una flexión de } \\
\text { cadera y recepciona } \\
\text { y lanza el balón.. }\end{array}$ & $\begin{array}{l}\text { Realiza el } \\
\text { movimiento } \\
\text { durante } \\
\text { 45segundos } \\
\text { Recepciona y } \\
\text { lanza el balón con } \\
\text { el brazo } \\
\text { contralateral a la } \\
\text { pierna de apoyo } \\
2 \text { series por cada } \\
\text { lado }\end{array}$ & Ojos abiertos & $\begin{array}{l}\text { Plana - } \\
\text { Regular }\end{array}$ \\
\hline $\begin{array}{l}\text { Apoyo unipodal } \\
\text { (Extensión de } \\
\text { cadera) } \\
\text { Recepción del } \\
\text { balón (42) }\end{array}$ & $\begin{array}{l}\text { El deportista } \\
\text { mantiene la posición } \\
\text { con apoyo unipodal } \\
\text { y con el miembro } \\
\text { contralateral realiza } \\
\text { una extensión de } \\
\text { cadera a la vez que } \\
\text { recepciona el balón }\end{array}$ & 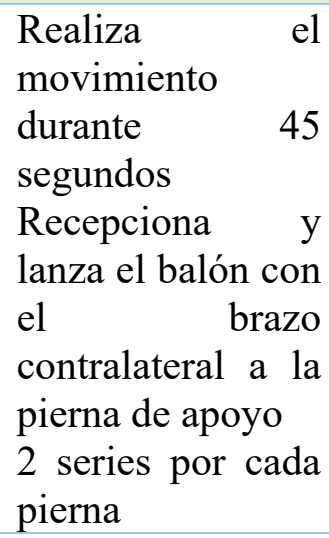 & Ojos abiertos & $\begin{array}{l}\text { Plana - } \\
\text { Regular }\end{array}$ \\
\hline
\end{tabular}




\begin{tabular}{|c|c|c|c|c|}
\hline \begin{tabular}{lr}
\multicolumn{2}{l}{ Apoyo unipodal } \\
(Flexión & de \\
tronco & $y$ \\
abducción & de \\
brazos) (42) &
\end{tabular} & $\begin{array}{l}\text { El deportista } \\
\text { mantiene el apoyo } \\
\text { unipodal y flexiona } \\
\text { lentamente el tronco } \\
\text { y abduce los brazos }\end{array}$ & $\begin{array}{l}\text { Realiza el } \\
\text { ejercicios durante } \\
45 \text { segundos } \\
2 \text { series por cada } \\
\text { pierna }\end{array}$ & Ojos abiertos & $\begin{array}{l}\text { Plana } \\
\text { Regular }\end{array}$ \\
\hline
\end{tabular}

\section{SESIONES 3 Y 4 (NIVEL 2)}

\begin{tabular}{|c|c|c|c|c|}
\hline EJERCICIOS & DESCRIPCIÓN & TIEMPO & $\begin{array}{l}\text { INFORMACI } \\
\text { ÓN VISUAL }\end{array}$ & $\begin{array}{l}\text { TIPO DE } \\
\text { SUPERFI } \\
\text { CIE }\end{array}$ \\
\hline $\begin{array}{l}\text { Apoyo unipodal } \\
\text { (Flexión de } \\
\text { cadera) (42) }\end{array}$ & $\begin{array}{l}\text { El deportista } \\
\text { mantiene la posición } \\
\text { sobre un pie y } \\
\text { rodilla extendida y } \\
\text { con la pierna } \\
\text { contralateral realiza } \\
\text { flexiones de cadera }\end{array}$ & $\begin{array}{l}\text { - Mantiene la posición } \\
\text { y realiza el } \\
\text { movimiento de flexión } \\
\text { durante } 45 \text { segundos } \\
\text { por cada lado } \\
2 \text { Series por cada } \\
\text { pierna }\end{array}$ & $\begin{array}{l}\text { Ojos abiertos } \\
\text { (Sesión 3) } \\
\text { Ojos cerrados ( } \\
\text { Sesión 4) }\end{array}$ & $\begin{array}{l}\text { Stability } \\
\text { Trainner } \\
\text { VERDE }\end{array}$ \\
\hline $\begin{array}{l}\text { Apoyo unipodal } \\
\text { (Extensión de } \\
\text { cadera) }(42)\end{array}$ & $\begin{array}{l}\text { El deportista } \\
\text { mantiene la posición } \\
\text { bípedo con apoyo de } \\
\text { un solo pie y con el } \\
\text { miembro } \\
\text { contralateral realiza } \\
\text { una extensión de } \\
\text { cadera }\end{array}$ & $\begin{array}{l}\text { Mantiene la posición y } \\
\text { realiza el movimiento } \\
\text { durante } 45 \text { segundos } \\
2 \text { Series por cada } \\
\text { pierna }\end{array}$ & $\begin{array}{l}\text { Ojos abiertos } \\
\text { (Sesión 3) } \\
\text { Ojos cerrados } \\
\text { (Sesión } 4)\end{array}$ & $\begin{array}{l}\text { Stability } \\
\text { Trainner } \\
\text { VERDE }\end{array}$ \\
\hline $\begin{array}{l}\text { Apoyo unipodal } \\
\text { (Flexión de } \\
\text { cadera) } \\
\text { Recepción del } \\
\text { balón } \\
(42)\end{array}$ & $\begin{array}{l}\text { El deportista se para } \\
\text { sobre un pie y con la } \\
\text { rodilla extendida y } \\
\text { con la pierna } \\
\text { contralateral realiza } \\
\text { una flexión de } \\
\text { cadera y recepciona } \\
\text { y lanza el balón. }\end{array}$ & $\begin{array}{l}\text { Realiza el movimiento } \\
\text { durante } 45 \text { segundos } \\
\text { Recepciona y lanza el } \\
\text { balón con el brazo } \\
\text { contralateral a la } \\
\text { pierna de apoyo } \\
2 \text { series por cada } \\
\text { pierna }\end{array}$ & Ojos abiertos & $\begin{array}{l}\text { Stability } \\
\text { Trainner } \\
\text { AZUL }\end{array}$ \\
\hline
\end{tabular}




\begin{tabular}{|c|c|c|c|c|}
\hline $\begin{array}{l}\text { Apoyo unipodal } \\
\text { ( Extensión de } \\
\text { cadera) } \\
\text { Recepción del } \\
\text { balón (42) }\end{array}$ & $\begin{array}{l}\text { El deportista } \\
\text { mantiene la posición } \\
\text { con apoyo unipodal } \\
\text { y con el miembro } \\
\text { contralateral realiza } \\
\text { una extensión de } \\
\text { cadera a la vez que } \\
\text { recepciona el balón }\end{array}$ & $\begin{array}{l}\text { Realiza el movimiento } \\
\text { durante } 45 \text { segundos } \\
\text { Recepciona y lanza el } \\
\text { balón con el brazo } \\
\text { contralateral a la } \\
\text { pierna de apoyo } \\
2 \text { series por cada } \\
\text { pierna }\end{array}$ & Ojos abiertos & $\begin{array}{l}\text { Stability } \\
\text { Trainner } \\
\text { AZUL }\end{array}$ \\
\hline $\begin{array}{lr}\text { Apoyo unipodal } \\
\text { ( Flexión de } \\
\text { tronco } & y \\
\text { abducción } & \text { de } \\
\text { brazos) }(42) & \end{array}$ & $\begin{array}{l}\text { El deportista } \\
\text { mantiene el apoyo } \\
\text { unipodal y flexiona } \\
\text { lentamente el tronco } \\
\text { y abduce los brazos }\end{array}$ & $\begin{array}{l}\text { Realiza el ejercicios } \\
\text { durante } 45 \text { segundos } \\
2 \text { series por cada } \\
\text { pierna }\end{array}$ & Ojos abiertos & $\begin{array}{l}\text { Stabily } \\
\text { Trainner } \\
\text { AZUL }\end{array}$ \\
\hline
\end{tabular}

SESIONES 5 y 6 (NIVEL 3)

\begin{tabular}{|c|c|c|c|c|}
\hline EJERCICIOS & DESCRIPCIÓN & TIEMPO & $\begin{array}{l}\text { INFORMACI } \\
\text { ÓN VISUAL }\end{array}$ & $\begin{array}{l}\text { TIPO DE } \\
\text { SUPERFI } \\
\text { CIE }\end{array}$ \\
\hline $\begin{array}{l}\text { Apoyo unipodal } \\
\text { (Flexión de } \\
\text { cadera) (42) }\end{array}$ & $\begin{array}{l}\text { El deportista } \\
\text { mantiene la posición } \\
\text { sobre un pie y } \\
\text { rodilla extendida y } \\
\text { con la pierna } \\
\text { contralateral realiza } \\
\text { flexiones de cadera }\end{array}$ & $\begin{array}{l}\text { - Mantiene la posición } \\
\text { y realiza el } \\
\text { movimiento de flexión } \\
\text { durante } 45 \text { segundos } \\
\text { por cada lado } \\
2 \text { Series por cada } \\
\text { pierna }\end{array}$ & $\begin{array}{l}\text { Ojos abiertos } \\
\text { (Sesión 5) } \\
\text { Ojos cerrados } \\
\text { (Sesión 6) }\end{array}$ & $\begin{array}{l}\text { Stability } \\
\text { Trainner } \\
\text { AZUL }\end{array}$ \\
\hline $\begin{array}{l}\text { Apoyo unipodal } \\
\text { (Extensión de } \\
\text { cadera) }(42)\end{array}$ & $\begin{array}{l}\text { El deportista } \\
\text { mantiene la posición } \\
\text { bípedo con apoyo de } \\
\text { un solo pie y con el } \\
\text { miembro } \\
\text { contralateral realiza } \\
\text { una extensión de } \\
\text { cadera }\end{array}$ & $\begin{array}{l}\text { Mantiene la posición y } \\
\text { realiza el movimiento } \\
\text { durante } 45 \text { segundos } \\
2 \text { Series por cada } \\
\text { pierna }\end{array}$ & $\begin{array}{l}\text { Ojos abiertos } \\
\text { (Sesión 5) } \\
\text { Ojos cerrados ( } \\
\text { Sesión 6) }\end{array}$ & $\begin{array}{l}\text { Stability } \\
\text { Trainner } \\
\text { AZUL }\end{array}$ \\
\hline
\end{tabular}




\begin{tabular}{|c|c|c|c|c|}
\hline $\begin{array}{l}\text { Apoyo unipodal } \\
\text { (Flexión de } \\
\text { cadera) } \\
\text { Recepción del } \\
\text { balón } \\
(42)\end{array}$ & $\begin{array}{l}\text { El deportista se para } \\
\text { sobre un pie y con la } \\
\text { rodilla extendida y } \\
\text { con la pierna } \\
\text { contralateral realiza } \\
\text { una flexión de } \\
\text { cadera y recepciona } \\
\text { y lanza el balón. }\end{array}$ & $\begin{array}{l}\text { Realiza el movimiento } \\
\text { durante } 45 \text { segundos } \\
\text { Recepciona y lanza el } \\
\text { balón con el brazo } \\
\text { contralateral a la } \\
\text { pierna de apoyo } \\
2 \text { series por cada } \\
\text { pierna }\end{array}$ & Ojos abiertos & $\begin{array}{l}\text { Stability } \\
\text { Trainner } \\
\text { AZUL }\end{array}$ \\
\hline $\begin{array}{l}\text { Apoyo unipodal } \\
\text { ( Extensión de } \\
\text { cadera) } \\
\text { Recepción del } \\
\text { balón (42) }\end{array}$ & $\begin{array}{l}\text { El deportista } \\
\text { mantiene la posición } \\
\text { con apoyo unipodal } \\
\text { y con el miembro } \\
\text { contralateral realiza } \\
\text { una extensión de } \\
\text { cadera a la vez que } \\
\text { recepciona el balón }\end{array}$ & $\begin{array}{l}\text { Realiza el movimiento } \\
\text { durante } 45 \text { segundos } \\
\text { Recepciona y lanza el } \\
\text { balón con el brazo } \\
\text { contralateral a la } \\
\text { pierna de apoyo } \\
2 \text { series por cada } \\
\text { pierna }\end{array}$ & Ojos abiertos & $\begin{array}{l}\text { Rocket } \\
\text { Board }\end{array}$ \\
\hline $\begin{array}{l}\text { Apoyo unipodal } \\
\text { ( Flexión de } \\
\text { tronco } \\
\text { abducción } \\
\text { brazos) (42) }\end{array}$ & $\begin{array}{l}\text { El deportista } \\
\text { mantiene el apoyo } \\
\text { unipodal y flexiona } \\
\text { lentamente el tronco } \\
\text { y abduce los brazos }\end{array}$ & $\begin{array}{l}\text { Realiza el ejercicios } \\
\text { durante } 45 \text { segundos } \\
2 \text { series por cada } \\
\text { pierna }\end{array}$ & Ojos abiertos & $\begin{array}{l}\text { Rocket } \\
\text { board }\end{array}$ \\
\hline
\end{tabular}


Anexo 2

\section{CONSENTIMIENTO INFORMADO}

A través de este documento se le invita a participar en un estudio de investigación acerca del efecto de un programa de propiocepción para el aumento del balance dinámico en los deportistas de las selecciones de vóley, futsal y básquet (damas y varones correspondientemente) de la Universidad Peruana de Ciencias Aplicadas.

Se le detallará en el siguiente escrito acerca de los objetivos, procedimientos, beneficios y riesgos de su participación durante el desarrollo de la investigación. Todo esto con la finalidad de confirmar su participación en el estudio.

\section{OBJETIVOS Y BENEFICIOS}

El objetivo del siguiente estudio es evaluar el aumento en el balance dinámico con la aplicación de un programa de propiocepción durante los entrenamientos.

El balance dinámico tiene un papel importante durante el movimiento, más aún en los deportes. Además, la propiocepción es la consciencia de la propia postura corporal y participa en la obtención de información del entorno que permite tener un equilibrio al realizar tareas determinadas. Los programas de propiocepción ayudan a prevenir lesiones deportivas, además de mejorar el balance dinámico. Por lo tanto, los resultados de este estudio pueden ayudar a incorporar un programa de propiocepción dentro del entrenamiento y obtener así un mejor desempeño en los deportistas.

\section{PROCEDIMIENTOS}

Se le hará la entrega de una encuesta basal conformada por 13 preguntas, que tiene como objetivo obtener información como: Edad, deporte, horas de práctica, etc.

Luego participará en la evaluación del balance dinámico que se realizará utilizando la herramienta Star Excursion Balance Test (SEBT) en la que se mantendrá parado en un solo pie (apoyo unipodal) y realizará movimientos en 8 direcciones diferentes con el pie contrario. Proceso para el cual los evaluadores han sido capacitados previamente.

Luego de obtenidos los datos del balance dinámico, se llevará a cabo el desarrollo del programa de propiocepción con una duración de 4 semanas, 3 veces por semana y durante 10 - 15 minutos por día. El programa ha sido previamente analizado por un especialista en Fisioterapia Deportiva, con la finalidad de obtener los mejores resultados 


\section{CONSIDERACIONES}

Si sufre alguna lesión durante el transcurso de las 4 semanas del desarrollo del programa, será retirado del mismo con la finalidad de conservar su bienestar físico. No todas las personas responden de la misma manera a los entrenamientos propioceptivos, por lo que algunos participantes les resultarán mucho más sencillo de realizarlos y a otros les tomará un poco más de tiempo.

\section{$\underline{\text { RIESGOS }}$}

La probabilidad de riesgo es baja, posiblemente la incomodidad durante algunos de los ejercicios propioceptivos, ya que estos requieren de movimientos que suelen evocar estructuras estabilizadoras y crear desequilibrio.

\section{AFIRMACIÓN DEL PARTICIPANTE}

Se me ha explicado de manera clara acerca de la investigación; por lo cual he decidido participar. Si tuviera alguna duda o pregunta en relación al desarrollo del programa puede comunicarse con: Enrique Zamora Gonzales (u201313514@upc.edu.pe), Rodrigo Palza Linares (u201322639@upc.edu.pe), Lic. Giancarlo Becerra Bravo (Giancarlo.becerra@upc.edu.pe), Heidi Kosakowski (pctfhkos@upc.edu.pe) Sulays Arias Mostacero (sulays.arias@upc.edu.pe).

Nombre del participante:

Firma del participante:

Nombre del Evaluador:

Firma del evaluador:

Lima, de del 2017 
